\title{
Nipen Saikia
}

\section{New theta-function identities and general theorems for the explicit evaluations of Ramanujan's continued fractions}

Received: 8 November 2014 / Accepted: 20 July 2016 / Published online: 2 August 2016

(C) The Author(s) 2016. This article is published with open access at Springerlink.com

\begin{abstract}
We prove some new theta-function identities for two continued fractions of Ramanujan which are analogous to those of Ramanujan-Göllnitz-Gordon continued fraction. Then these identities are used to prove new general theorems for the explicit evaluations of the continued fractions.
\end{abstract}

Mathematics Subject Classification 30B70 - 33D15 - 33D90 - 11F20

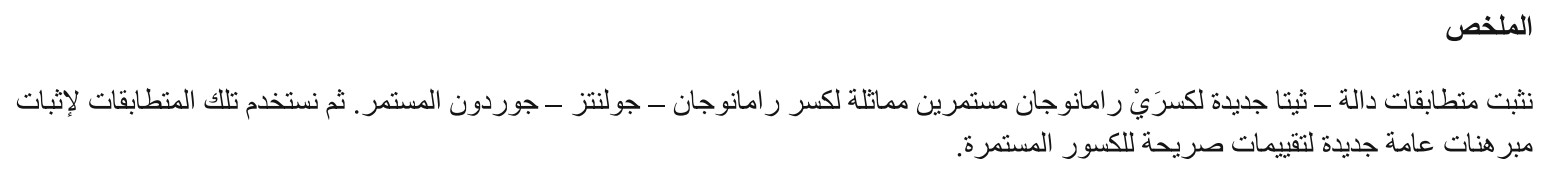

\section{Introduction}

Throughout the paper, we assume $|q|<1$ and

$$
(a ; q)_{\infty}:=\prod_{n=0}^{\infty}\left(1-a q^{n}\right) .
$$

Ramanujan's general theta-function $f(a, b)$ is given by

$$
f(a, b)=\sum_{k=-\infty}^{\infty} a^{k(k+1) / 2} b^{k(k-1) / 2}, \quad|a b|<1 .
$$

Three special cases of $f(a, b)$ are

$$
\begin{aligned}
& \phi(q):=f(q, q)=\sum_{n=-\infty}^{\infty} q^{n^{2}}=\frac{\left(-q ; q^{2}\right)_{\infty}\left(q^{2} ; q^{2}\right)_{\infty}}{\left(q ; q^{2}\right)_{\infty}\left(-q^{2} ; q^{2}\right)_{\infty}} \\
& \psi(q):=f\left(q, q^{3}\right)=\sum_{k=0}^{\infty} q^{k(k+1) / 2}=\frac{\left(q^{2} ; q^{2}\right)_{\infty}}{\left(q ; q^{2}\right)_{\infty}}
\end{aligned}
$$

and

$$
f(-q):=f\left(-q,-q^{2}\right)=\sum_{n=-\infty}^{\infty}(-1)^{n} q^{n(3 n-1) / 2}=(q ; q)_{\infty}
$$

N. Saikia $(\varangle)$

Department of Mathematics, Rajiv Gandhi University, Rono Hills, Doimukh 791112, Papum Pare, Arunachal Pradesh, India E-mail: nipennak@yahoo.com 
In his notebooks, Ramanujan recorded several $q$-continued fractions which have beautiful theories. Among them is the celebrated Ramanujan-Göllnitz-Gordon continued fraction $K(q)$ defined by

$$
K(q):=\frac{q^{1 / 2}}{1+q+\frac{q^{2}}{1+q^{3}+\frac{q^{4}}{1+q^{5}+\cdots}}},|q|<1 .
$$

On page 299 of his second notebook [11], Ramanujan recorded a product representation of $K(q)$, namely

$$
K(q):=q^{1 / 2} \frac{\left(q ; q^{8}\right)_{\infty}\left(q^{7} ; q^{8}\right)_{\infty}}{\left(q^{3} ; q^{8}\right)_{\infty}\left(q^{5} ; q^{8}\right)_{\infty}} .
$$

Without the knowledge of Ramanujan's work, Göllnitz [8] and Gordon [9], independently, rediscovered and proved (1.6). Shortly thereafter, Andrews [1] proved (1.6) as a corollary of a more general result. Ramanathan [10] also found an alternative proof of (1.6). In addition to (1.6), Ramanujan offered the following two other identities [11, p. 299] for $K(q)$ :

$$
\frac{1}{K(q)}-K(q)=\frac{\phi\left(q^{2}\right)}{q^{1 / 2} \psi\left(q^{4}\right)}
$$

and

$$
\frac{1}{K(q)}+K(q)=\frac{\phi(q)}{q^{1 / 2} \psi\left(q^{4}\right)} .
$$

Proofs of (1.7) and (1.8) can be found in [7] and [12]. Yuttanan [19] also proved that

$$
\frac{1}{K(q)}+2-K(q)=\frac{\phi\left(q^{1 / 2}\right)}{q^{1 / 2} \psi\left(q^{4}\right)}
$$

and

$$
\frac{1}{K(q)}-2-K(q)=\frac{\phi\left(-q^{1 / 2}\right)}{q^{1 / 2} \psi\left(q^{4}\right)}
$$

For further references on $K(q)$ see Chan and Huang [7], Vasuki and Kumar [18], and Baruah and Saikia [3].

In this paper, we prove some theta-function identities analogous to (1.7)-(1.10) for the continued fractions $T(q)$ and $W(q)$ which are defined, respectively, as

$$
T(q):=\frac{q}{1-q^{2}+\frac{q^{4}}{1-q^{6}+\frac{q^{8}}{1-q^{10}+\cdots}}}, \quad|q|<1 .
$$

and

$$
W(q):=\frac{q}{1-q^{2}+\frac{q^{2}\left(1+q^{2}\right)^{2}}{1-q^{6}+\frac{q^{4}\left(1+q^{4}\right)^{2}}{1-q^{10}+\cdots}}}, \quad|q|<1 .
$$

and use them to prove new general theorems for the explicit evaluations of $T(q)$ and $W(q)$. The continued fractions $T(q)$ and $W(q)$ are introduced and studied by Saikia in [14] and [15], respectively. Saikia [14, p. 4, Theorem 3.1] proved that

$$
T(q)=\frac{f(q)-f(-q)}{f(q)+f(-q)} .
$$

The identity analogous to (1.13) and satisfied by the continued fraction $W(q)$ is [15, Theorem 3.1]:

$$
2 W(q)=\frac{f^{2}(q)-f^{2}(-q)}{f^{2}(q)+f^{2}(-q)} .
$$


Saikia also established some modular relations and explicit values for $T(q)$ and $W(q)$ in [14] and [15], respectively.

In Sects. 3 and 4, we prove new theta-function identities for the continued fractions $T(q)$ and $W(q)$, respectively. In Sects. 5 and 6, we prove new general theorems for the explicit evaluations of $T(q)$ and $W(q)$ by using theta-function identities established in Sects. 3 and 4, respectively, and give examples of explicit evaluations. Section 2 is devoted to record some preliminary results for ready references in this paper.

To end this introduction, we define some parameters of theta-functions which will be used in the explicit evaluations of $T(q)$ and $W(q)$. For any positive real numbers $k$ and $n$, define

$$
\begin{aligned}
& A_{k, n}=\frac{\phi(-q)}{2 k^{1 / 4} q^{k / 4} \psi\left(q^{2 k}\right)} ; \quad q=\mathrm{e}^{-\pi \sqrt{n / k}}, \\
& s_{4, n}=\frac{f(q)}{\sqrt{2} q^{1 / 8} f\left(-q^{4}\right)} ; \quad q=\mathrm{e}^{-\pi \sqrt{n} / 2}, \\
& J_{n}=\frac{f(-q)}{\sqrt{2} q^{1 / 8} f\left(-q^{4}\right)} ; \quad q=\mathrm{e}^{-\pi \sqrt{n}} .
\end{aligned}
$$

The parameter $A_{k, n}$ is introduced by Saikia [13, p. 107, (1.7)]. The parameter $s_{4, n}$ is the particular case $k=4$ of the general parameter $s_{k, n}$ defined by

$$
s_{k, n}=\frac{f(q)}{k^{1 / 4} q^{(k-1) / 24} f\left(-(-1)^{k} q^{k}\right)} ; \quad q=\mathrm{e}^{-\pi \sqrt{n / k}}
$$

and is due to Berndt $\left[6\right.$, p. 9, (4.7)]. The parameter $J_{n}$ is the particular case $k=4$ of the general parameter $r_{k, n}$, introduced by Yi [20, p. 11, (2.1.1)] (also see [6, p. 9, (4.6)]) and defined by

$$
r_{k, n}:=\frac{f(-q)}{k^{1 / 4} q^{(k-1) / 24} f\left(-q^{k}\right)}, \quad q=\mathrm{e}^{-2 \pi \sqrt{n / k}} .
$$

Yi [20] evaluated several explicit values of the parameter $r_{k, n}$.

\section{Preliminary results}

This section is devoted to record some transformation formulas and $P-Q$ theta-function identities which will be used in the succeeding sections. The $P-Q$ identities presented in Lemmas 2.6-2.8 are new. Since modular equations are key in the proofs of $P-Q$ theta-function identities, first we define Ramanujan's modular equation.

The ordinary or Gaussian hypergeometric function ${ }_{2} F_{1}(a, b ; c ; x)$ is defined by

$$
{ }_{2} F_{1}(a, b ; c ; x)=\sum_{n=0}^{\infty} \frac{(a)_{n}(b)_{n}}{(c)_{n} n !} x^{n},
$$

where $(a)_{0}=1$ and $(a)_{n}=a(a+1)(a+2) \cdots a(a+n-1)$ for $n \geq 1$ and $|x|<1$.

Let, for $0<\alpha<1$,

$$
z_{1}={ }_{2} F_{1}\left(\frac{1}{2}, \frac{1}{2} ; 1 ; \alpha\right) \text { and } q=\exp \left(-\pi \csc (\pi / 2) \frac{{ }_{2} F_{1}\left(\frac{1}{2}, \frac{1}{2} ; 1 ; 1-\alpha\right)}{{ }_{2} F_{1}\left(\frac{1}{2}, \frac{1}{2} ; 1 ; \alpha\right)}\right) .
$$

Assume that for some integer $n$

$$
n \frac{{ }_{2} F_{1}\left(\frac{1}{2}, \frac{1}{2} ; 1 ; 1-\alpha\right)}{{ }_{2} F_{1}\left(\frac{1}{2}, \frac{1}{2} ; 1 ; \alpha\right)}=\frac{{ }_{2} F_{1}\left(\frac{1}{2}, \frac{1}{2} ; 1 ; 1-\beta\right)}{{ }_{2} F_{1}\left(\frac{1}{2}, \frac{1}{2} ; 1 ; \beta\right)} .
$$

Then a modular equation of degree $n$ is a relation between $\alpha$ and $\beta$ induced by (2.2). We often say $\beta$ has degree $n$ over $\alpha$. The multiplier $m$ connecting $\alpha$ and $\beta$ is defined by $m=z_{1} / z_{n}$, where $z_{n}={ }_{2} F_{1}\left(\frac{1}{2}, \frac{1}{2} ; 1 ; \beta\right)$. 
Lemma 2.1 [13, p. 111, Theorem 4.1] We have

$$
A_{k, 1}=1 \text { and } A_{k, 1 / n}=1 / A_{k, n} .
$$

Lemma 2.2 [2, p. 9, Theorem 6.1] We have

$$
J_{1 / n}=1 / J_{n} .
$$

Lemma 2.3 [5, p. 40, Entry 25] We have

$$
\begin{gathered}
\phi(q)+\phi(-q)=2 \phi\left(q^{4}\right), \\
\phi(q)-\phi(-q)=4 q \psi\left(q^{8}\right), \\
\phi^{2}(q)-\phi^{2}(-q)=8 q \psi^{2}\left(q^{4}\right), \\
\phi^{2}(q)+\phi^{2}(-q)=2 \phi^{2}\left(q^{2}\right), \\
\phi(q) \phi(-q)=\phi^{2}\left(-q^{2}\right) .
\end{gathered}
$$

Lemma 2.4 [4, Lemmas 3.10 and 3.11] We have

$$
\begin{gathered}
\phi(q)=\frac{f^{5}\left(-q^{2}\right)}{f^{2}(-q) f^{2}\left(-q^{4}\right)}, \quad \phi(-q)=\frac{f^{2}(-q)}{f\left(-q^{2}\right)}, \\
\psi(q)=\frac{f^{2}\left(-q^{2}\right)}{f(-q)}, \quad f(q)=\frac{f^{3}\left(-q^{2}\right)}{f(-q) f\left(-q^{4}\right)} .
\end{gathered}
$$

Lemma 2.5 [5, p. 122-123, Entry 10(ii) and Entry 11(v)] If $z_{1}, q$, and $\alpha$ are related by (2.1), then

$$
\phi(-q)=\sqrt{z_{1}}(1-\alpha)^{1 / 4} \quad \text { and } \quad \psi\left(q^{8}\right)=\frac{1}{4} \sqrt{z_{1}}\left\{1-(1-\alpha)^{1 / 4}\right\} / q .
$$

Lemma 2.6 If $P=\frac{\phi(-q)}{q \psi\left(q^{8}\right)}$ and $Q=\frac{\phi\left(-q^{2}\right)}{q^{2} \psi\left(q^{16}\right)}$,

$$
\text { then } Q^{2}-P^{2} Q-4 P Q-2 P^{2}-8 P=0 .
$$

Proof Transcribing using Lemma 2.5, we find that

$$
(1-\alpha)^{1 / 4}=\frac{P}{4+P} \quad \text { and } \quad \beta=1-\left(\frac{Q}{4+Q}\right)^{4},
$$

where $\beta$ has degree 2 over $\alpha$. From [5, p. 214, Entry 24(iii)] we note that, if $\beta$ has degree 2 over $\alpha$, then

$$
m \sqrt{1-\alpha}+\sqrt{\beta}=1
$$

and

$$
m^{2} \sqrt{1-\alpha}+\beta=1 .
$$

Eliminating $m$ between (2.10) and (2.11) and then simplifying, we deduce that

$$
(1+\beta+(\beta-1) \sqrt{1-\alpha})^{2}-4 \beta=0 .
$$

Employing (2.9) in (2.12) and factorizing using Mathematica, we obtain

$$
\begin{aligned}
& \left(Q^{2}-P^{2} Q-4 P Q-2 P^{2}-8 P\right) \\
& \quad \times\left(32 P+8 P^{2}+16 P Q+4 P^{2} Q+4 Q^{2}+4 P Q^{2}+P^{2} Q^{2}\right)=0 .
\end{aligned}
$$

Since the second factor is non-zero, we arrive at the desired result. 
Lemma 2.7 If $P=\frac{\phi(-q)}{q \psi\left(q^{8}\right)}$ and $Q=\frac{\phi\left(-q^{3}\right)}{q^{3} \psi\left(q^{24}\right)}$

$$
\begin{aligned}
& \text { then } P^{4}-64 P Q-48 P^{2} Q-12 P^{3} Q-48 P Q^{2}-30 P^{2} Q^{2}-6 P^{3} Q^{2}-12 P Q^{3}-6 P^{2} Q^{3} \\
& -P^{3} Q^{3}+Q^{4}=0 .
\end{aligned}
$$

Proof Transcribing using Lemma 2.5, we find that

$$
\begin{gathered}
(1-\alpha)^{1 / 4}=\frac{P}{4+P} \quad(1-\beta)^{1 / 4}=\frac{Q}{4+Q}, \\
\alpha=1-\left(\frac{P}{4+P}\right)^{4}, \quad \text { and } \quad \beta=1-\left(\frac{Q}{4+Q}\right)^{4},
\end{gathered}
$$

where $\beta$ has degree 3 over $\alpha$. From [5, p. 230, Entry 5(ii)] we note that, if $\beta$ has degree 3 over $\alpha$, then

$$
(\alpha \beta)^{1 / 4}+\{(1-\alpha)(1-\beta)\}^{1 / 4}=1
$$

and can also be expressed as

$$
\alpha \beta-\left(1-(1-\alpha)^{1 / 4}(1-\beta)^{1 / 4}\right)^{4}=0 .
$$

Employing (2.15) and (2.16) in (2.18) and simplifying with the help of Mathematica, we arrive at the desired result.

Lemma 2.8 If $P=\frac{\phi(-q)}{q \psi\left(q^{8}\right)}$ and $Q=\frac{\phi\left(-q^{5}\right)}{q^{5} \psi\left(q^{40}\right)}$,

$$
\begin{aligned}
& \text { then } P^{6}-4096 P Q-5120 P^{2} Q-2560 P^{3} Q-640 P^{4} Q-70 P^{5} Q-5120 P Q^{2} \\
& -6400 P^{2} Q^{2}-3200 P^{3} Q^{2}-785 P^{4} Q^{2}-80 P^{5} Q^{2}-2560 P Q^{3}-3200 P^{2} Q^{3}-1620 P^{3} Q^{3} \\
& -400 P^{4} Q^{3}-40 P^{5} Q^{3}-640 P Q^{4}-785 P^{2} Q^{4}-400 P^{3} Q^{4}-100 P^{4} Q^{4}-10 P^{5} Q^{4}-70 P Q^{5} \\
& -80 P^{2} Q^{5}-40 P^{3} Q^{5}-10 P^{4} Q^{5}-P^{5} Q^{5}+Q^{6}=0 .
\end{aligned}
$$

Proof Transcribing using Lemma 2.5, we find that

$$
c:=(1-\alpha)^{1 / 8}=\sqrt{\frac{P}{4+P}} \quad d:=(1-\beta)^{1 / 8}=\sqrt{\frac{Q}{4+Q}},
$$

where $\beta$ has degree 5 over $\alpha$. From [5, p. 280-281, Entry 13(v) \& (vi)] we note that, if $\beta$ has degree 5 over $\alpha$, then

$$
\begin{aligned}
& m=\frac{1+\left(\frac{(1-\beta)^{5}}{1-\alpha}\right)^{1 / 8}}{1+\left\{(1-\alpha)^{3}(1-\beta)\right\}^{1 / 8}} \\
& \frac{5}{m}=\frac{1-\left(\frac{(1-\alpha)^{5}}{1-\beta}\right)^{1 / 8}}{1-\left\{(1-\alpha)(1-\beta)^{3}\right\}^{1 / 8}}
\end{aligned}
$$

Employing (2.20) in (2.21) and (2.22), we find that

$$
m=\frac{c+d^{5}}{c\left(1+c^{3} d\right)}
$$


and

$$
\frac{5}{m}=\frac{d-c^{5}}{d\left(1-c d^{3}\right)}
$$

respectively. Eliminating $m$ between (2.23) and (2.24) and simplifying, we deduce that

$$
5 c d\left(1+c^{3} d\right)\left(1-c d^{3}\right)-\left(c+d^{5}\right)\left(d-c^{5}\right)=0 .
$$

Equivalently,

$$
4\left(c d-c^{5} d^{5}\right)=\left(5 c^{2} d^{4}-5 c^{4} d^{2}-c^{6}+d^{6}\right) .
$$

Squaring (2.26) and substituting for $c$ and $d$ from (2.20) and simplifying with the help of Mathematica, we arrive at desired result.

\section{New identities for $T(q)$}

In this section we prove theta-function identities for $T(q)$ analogous to (1.7)-(1.10).

Theorem 3.1 We have

$$
\begin{aligned}
\text { (i) } \frac{1}{T\left(q^{1 / 4}\right)}+T\left(q^{1 / 4}\right) & =\frac{\phi(q)}{q^{1 / 4} \psi\left(q^{2}\right)}=\frac{f^{2}(q)}{q^{1 / 4} f^{2}\left(-q^{4}\right)}, \\
\text { (ii) } \frac{1}{T\left(q^{1 / 2}\right)}-T\left(q^{1 / 2}\right) & =\frac{\phi(-q)}{q^{1 / 2} \psi\left(q^{4}\right)} \\
\text { (iii) } \frac{1}{T(q)}+2+T(q) & =\frac{\phi(q)}{q \psi\left(q^{8}\right)} \\
\text { (iv) } \frac{1}{T(q)}-2+T(q) & =\frac{\phi(-q)}{q \psi\left(q^{8}\right)} .
\end{aligned}
$$

Proof (i) From (1.13), we note that

$$
\begin{aligned}
\frac{1}{T(q)}+T(q) & =\frac{f(q)+f(-q)}{f(q)-f(-q)}+\frac{f(q)-f(-q)}{f(q)+f(-q)} \\
& =\frac{2\left\{f^{2}(q)+f^{2}(-q)\right\}}{f^{2}(q)-f^{2}(-q)} .
\end{aligned}
$$

From Lemma 2.4, we note that

$$
\begin{aligned}
f^{2}(q)=\frac{f^{6}\left(-q^{2}\right)}{f^{2}(-q) f^{2}\left(-q^{4}\right)} & =\left(\frac{f^{5}\left(-q^{2}\right)}{f^{2}(-q) f^{2}\left(-q^{4}\right)}\right)\left(\frac{f\left(-q^{2}\right)}{f^{2}(-q)}\right) f^{2}(-q) \\
& =\frac{\phi(q)}{\phi(-q)} f^{2}(-q) .
\end{aligned}
$$

Employing (3.2) in (3.1), and simplifying, we deduce that

$$
\frac{1}{T(q)}+T(q)=\frac{2\{\phi(q)+\phi(-q)\}}{\phi(q)-\phi(-q)} .
$$

Employing (2.3) and (2.4) in (3.3) and simplifying, we obtain

$$
\frac{1}{T(q)}+T(q)=\frac{\phi\left(q^{4}\right)}{q \psi\left(q^{8}\right)} .
$$

Replacing $q$ by $q^{1 / 4}$ in (3.4), we prove the first equality. To prove the second equality, from Lemma 2.4 we note that

$$
\frac{\phi(q)}{\psi\left(q^{2}\right)}=\frac{f^{2}(q)}{f^{2}\left(-q^{4}\right)} .
$$


Employing (3.5) in the first equality, we arrive at the desired result.

(ii) From (1.13), we deduce that

$$
\frac{1}{T(q)}-T(q)=\frac{4 f(q) f(-q)}{f^{2}(q)-f^{2}(-q)} .
$$

Employing (3.2) in (3.6) and simplifying, we find that

$$
\frac{1}{T(q)}-T(q)=4\left(\frac{f(q) \phi(-q)}{f(-q)}\right)\left(\frac{1}{\phi(q)-\phi(-q)}\right) .
$$

From Lemma 2.4, we note that

$$
\frac{f(q) \phi(-q)}{f(-q)}=\frac{f^{2}(-q)}{f\left(-q^{4}\right)}=\phi\left(-q^{2}\right) .
$$

Employing (2.4) and (3.8) in (3.7) and simplifying, we obtain

$$
\frac{1}{T(q)}-T(q)=\frac{\phi\left(-q^{2}\right)}{q \psi\left(q^{8}\right)} .
$$

Replacing $q$ by $q^{1 / 2}$ in (3.9), we arrive at the desired result.

(iii) From (1.13), we deduce that

$$
\begin{aligned}
\frac{1}{\sqrt{T(q)}}+\sqrt{T(q)} & =\sqrt{\frac{f(q)+f(-q)}{f(q)-f(-q)}}+\sqrt{\frac{f(q)-f(-q)}{f(q)+f(-q)}} \\
& =\frac{2 f(q)}{\sqrt{f^{2}(q)-f^{2}(-q)}} .
\end{aligned}
$$

Employing (3.2) in (3.10) and simplifying, we obtain

$$
\frac{1}{\sqrt{T(q)}}+\sqrt{T(q)}=\frac{2 f(q) \sqrt{\phi(-q)}}{f(-q) \sqrt{\phi(q)-\phi(-q)}} .
$$

Squaring (3.11), we find that

$$
\frac{1}{T(q)}+2+T(q)=\frac{4 f^{2}(q) \phi(-q)}{f^{2}(-q)\{\phi(q)-\phi(-q)\}}
$$

Employing (2.4) and (3.2) in (3.12) and simplifying, we arrive at the desired result.

(iv) From (1.13), we deduce that

$$
\begin{aligned}
\frac{1}{\sqrt{T(q)}}-\sqrt{T(q)} & =\sqrt{\frac{f(q)+f(-q)}{f(q)-f(-q)}}-\sqrt{\frac{f(q)-f(-q)}{f(q)+f(-q)}} \\
& =\frac{2 f(-q)}{\sqrt{f^{2}(q)-f^{2}(-q)}} .
\end{aligned}
$$

Squaring (3.13) and simplifying by employing (3.2), we obtain

$$
\frac{1}{T(q)}-2+T(q)=\frac{4 \phi(-q)}{\phi(q)-\phi(-q)} .
$$

Employing (2.4) in (3.14) and simplifying, we complete the proof. 
Corollary 3.2 We have

$$
\begin{aligned}
& \text { (i) } \frac{1}{T^{2}(q)}-2+T^{2}(q)=\frac{\phi^{2}\left(-q^{2}\right)}{q^{2} \psi^{2}\left(q^{8}\right)}, \\
& \text { (ii) } \frac{1}{\sqrt{T(q)}}+\sqrt{T(q)}=\sqrt{\frac{\phi(q)}{q \psi\left(q^{8}\right)}} \\
& \text { (iii) } \frac{1}{\sqrt{T(q)}}-\sqrt{T(q)}=\sqrt{\frac{\phi(-q)}{q \psi\left(q^{8}\right)}} .
\end{aligned}
$$

Proof From Theorem 3.1(iii) and (iv), we have

$$
\left(\frac{1}{T(q)}+2+T(q)\right)\left(\frac{1}{T(q)}-2+T(q)\right)=\frac{\phi(q) \phi(-q)}{q^{2} \psi^{2}\left(q^{8}\right)} .
$$

Employing (2.7) in (3.15), we arrive at (i). To prove (ii) and (iii), we employ (3.2) in (3.11) and (3.13), respectively, and simplify.

\section{New identities for $W(q)$}

This section is devoted to proving theta-function identities analogous to (1.7)-(1.10) for the continued fraction $W(q)$.

Theorem 4.1 We have

$$
\begin{aligned}
& \text { (i) } \frac{1}{W(\sqrt{q})}+4 W(\sqrt{q})=\frac{\phi^{2}(q)}{q^{1 / 2} \psi^{2}\left(q^{2}\right)}=\frac{f^{4}(q)}{q^{1 / 2} f^{4}\left(-q^{4}\right)}, \\
& \text { (ii) } \frac{1}{W(\sqrt{q})}-4 W(\sqrt{q})=\frac{\phi^{2}(-q)}{q^{1 / 2} \psi^{2}\left(q^{2}\right)}=\frac{f^{4}(-q)}{q^{1 / 2} f^{4}\left(-q^{4}\right)}, \\
& \text { (iii) } \frac{1}{W^{2}(\sqrt{q})}-16 W^{2}(\sqrt{q})=\frac{\phi^{4}\left(-q^{2}\right)}{q \psi^{4}\left(q^{2}\right)} .
\end{aligned}
$$

Proof (i) From (1.14), we deduce that

$$
\frac{1}{2 W(q)}+2 W(q)=\frac{2\left\{f^{4}(q)+f^{4}(-q)\right\}}{f^{4}(q)-f^{4}(-q)} .
$$

Squaring (3.2), then employing in (4.1) and simplifying, we obtain

$$
\frac{1}{2 W(q)}+2 W(q)=\frac{2\left\{\phi^{2}(q)+\phi^{2}(-q)\right\}}{\phi^{2}(q)-\phi^{2}(-q)} .
$$

Employing (2.5) and (2.6) in (4.2) and simplifying, we obtain

$$
\frac{1}{W(q)}+4 W(q)=\frac{\phi^{2}\left(q^{2}\right)}{q \psi^{2}\left(q^{4}\right)} .
$$

Replacing $q$ by $\sqrt{q}$ in (4.3) we prove the first equality. To prove the second equality, from Lemma 2.4 we note that

$$
\frac{\phi(q)}{\psi\left(q^{2}\right)}=\frac{f^{2}(q)}{f^{2}\left(-q^{4}\right)} .
$$

Employing (4.4) in the first equality, we arrive at the desired result.

(ii) From (1.14), we note that

$$
\frac{1}{2 W(q)}-2 W(q)=\frac{4 f^{2}(q) f^{2}(-q)}{f^{4}(q)-f^{4}(-q)} .
$$


Employing (3.2) in (4.5) and simplifying, we obtain

$$
\frac{1}{2 W(q)}+2 W(q)=\frac{4 \phi(q) \phi(-q)}{\phi^{2}(q)-\phi^{2}(-q)} .
$$

Employing (2.5) and (2.7) in (4.6) and simplifying, we obtain

$$
\frac{1}{W(q)}+4 W(q)=\frac{\phi^{2}\left(-q^{2}\right)}{q \psi^{2}\left(q^{4}\right)} .
$$

Replacing $q$ by $\sqrt{q}$ in (4.7), we prove the first equality. To prove the second equality, from Lemma 2.4 we note that

$$
\frac{\phi(-q)}{\psi\left(q^{2}\right)}=\frac{f^{2}(-q)}{f^{2}\left(-q^{4}\right)}
$$

Employing (4.8) in the first equality, we arrive at the desired result.

(iii) From part (i) and (ii), we deduce that

$$
\left(\frac{1}{W(\sqrt{q})}+4 W(\sqrt{q})\right)\left(\frac{1}{W(\sqrt{q})}-4 W(\sqrt{q})\right)=\frac{\phi^{2}(q) \phi^{2}(-q)}{q \psi^{4}\left(q^{2}\right)} .
$$

Employing (2.7) in (4.9) and simplifying, we complete the proof.

Theorem 4.2 We have

$$
\begin{aligned}
& \text { (i) } \frac{1}{\sqrt{W(q)}}+2 \sqrt{W(q)}=\frac{\phi(q)}{q^{1 / 2} \psi\left(q^{4}\right)}, \\
& \text { (ii) } \frac{1}{\sqrt{W(q)}}-2 \sqrt{W(q)}=\frac{\phi(-q)}{q^{1 / 2} \psi\left(q^{4}\right)} .
\end{aligned}
$$

Proof (i) From (1.14), we deduce that

$$
\frac{1}{\sqrt{2 W(q)}}+\sqrt{2 W(q)}=\frac{2 f^{2}(q)}{\sqrt{f^{4}(q)-f^{4}(-q)}} .
$$

Employing (3.2) in (4.10) and simplifying, we obtain

$$
\frac{1}{\sqrt{2 W(q)}}+\sqrt{2 W(q)}=\frac{2 \phi(q)}{\sqrt{\phi^{2}(q)-\phi^{2}(-q)}} .
$$

Employing (2.5) in (4.11) and simplifying, we arrive at the desired result.

(ii) From (1.14), we deduce that

$$
\frac{1}{\sqrt{2 W(q)}}-\sqrt{2 W(q)}=\frac{2 f^{2}(-q)}{\sqrt{f^{4}(q)-f^{4}(-q)}} .
$$

Employing (3.2) in (4.12) and simplifying, we obtain

$$
\frac{1}{\sqrt{2 W(q)}}-\sqrt{2 W(q)}=\frac{2 \phi(-q)}{\sqrt{\phi^{2}(q)-\phi^{2}(-q)}} .
$$

Employing (2.5) in (4.13) and simplifying, we complete the proof.

Corollary 4.3 We have

$$
\begin{aligned}
\text { (i) } \frac{1}{W(q)}+4+4 W(q) & =\frac{\phi^{2}(q)}{q \psi^{2}\left(q^{4}\right)}, \\
\text { (ii) } \frac{1}{W(q)}-4+4 W(q) & =\frac{\phi^{2}(-q)}{q \psi^{2}\left(q^{4}\right)} .
\end{aligned}
$$

Proof Squaring Theorem 4.2(i) and (ii) we easily arrive at (i) and (ii), respectively. 


\section{General theorems for explicit evaluations of $T(q)$}

In this section we prove new general theorems for the explicit evaluations of $T(q)$ and give examples.

Theorem 5.1 If $s_{4, n}$ is as defined in (1.16), then

$$
\frac{1}{T\left(e^{-\pi \sqrt{n} / 8}\right)}+T\left(e^{-\pi \sqrt{n} / 8}\right)=2 s_{4, n}^{2} .
$$

Proof Setting $q=\mathrm{e}^{-\pi \sqrt{n} / 2}$ in Theorem 3.1(i) and employing the definition of $s_{4, n}$ we complete the proof.

Remark 5.2 From Theorem 5.1 it is obvious that if we know explicit values of the parameter $s_{4, n}$ the explicit values of $T\left(e^{-\pi \sqrt{n} / 8}\right)$ can be easily evaluated. Baruah and Saikia [3] evaluated $s_{4, n}$ for $n=1,2,3,4,5,7,8$, $9,10,12,13,15,16,18,20,24,25,28,32,36,40,52,64,68,72,100,108,144,196,2 / 3,1 / 2,4 / 7,4 / 5,4 / 9,4 /$ $25,4 / 49,2 / 5,1 / 3,1 / 5,1 / 7,1 / 13,1 / 15$, and $1 / 25$. For example, setting $n=4$, employing the value $s_{4,4}=2^{1 / 8}$ in Theorem 5.1 and solving the resulting equation, we obtain

$$
T\left(\mathrm{e}^{-\pi / 4}\right)=2^{1 / 4} \pm \sqrt{-1+\sqrt{2}} .
$$

For $|q|<1$, neglecting $q^{8}$ and higher powers of $q$ in (1.11), we find that

$$
T(q) \approx q\left(1-q^{6}\right)\left(\left(1-q^{2}\right)\left(1-q^{6}\right)+q^{4}\right)^{-1}
$$

and for $q=e^{-\pi / 4}, T\left(e^{-\pi / 4}\right) \approx 0.545559<1$. So choosing minus sign in (5.1), we obtain

$$
T\left(e^{-\pi / 4}\right)=2^{1 / 4}-\sqrt{-1+\sqrt{2}}
$$

Theorem 5.3 We have

$$
\begin{aligned}
& \text { (i) } \frac{1}{T\left(e^{-\pi \sqrt{n} /(2 \sqrt{2})}\right)}-T\left(e^{-\pi \sqrt{n} /(2 \sqrt{2})}\right)=2^{5 / 4} A_{2, n}, \\
& \text { (ii) } \frac{1}{T\left(e^{-\pi /(2 \sqrt{2 n})}\right)}-T\left(e^{-\pi /(2 \sqrt{2 n})}\right)=\frac{2^{5 / 4}}{A_{2, n}} .
\end{aligned}
$$

Proof Setting $q=\mathrm{e}^{-\pi \sqrt{n / 2}}$ in Theorem 3.1(ii) and employing the definition of $A_{k, n}$ with $k=2$, we arrive at (i). Replacing $n$ by $1 / n$ in (i) and simplifying using Lemma 2.1, we complete the proof of (ii).

Remark 5.4 From Theorem 5.3(i) and (ii) it is clear that if we know explicit values of the parameter $A_{2, n}$ then explicit values of $T\left(e^{-\pi \sqrt{n} /(2 \sqrt{2})}\right)$ and $T\left(e^{-\pi /(2 \sqrt{2 n})}\right)$ can easily be evaluated, respectively. Saikia [13,17] evaluated $A_{2, n}$ for $n=1,2,3,4,5,7,9,25$, and 49. For example, setting $n=3$, employing the value $A_{2,3}=\sqrt{2+\sqrt{2}+\sqrt{9+6 \sqrt{2}}}$ from [13, p. 115, Theorem 5.4(i)] in Theorem 5.3(i) and (ii) and solving the resulting equations, we evaluate

$$
T\left(e^{-\pi \sqrt{3} /(2 \sqrt{2})}\right)=\sqrt{3+2 \sqrt{2}+\sqrt{18+12 \sqrt{2}}}-2^{1 / 4} \sqrt{2+\sqrt{2}+\sqrt{9+6 \sqrt{2}}}
$$

and

$$
T\left(e^{-\pi /(2 \sqrt{6})}\right)=\frac{-2^{1 / 4}+\sqrt{2+2 \sqrt{2}+\sqrt{9+6 \sqrt{2}}}}{\sqrt{2+\sqrt{2}+\sqrt{9+6 \sqrt{2}}}},
$$

respectively. 
Theorem 5.5 We have

$$
\begin{aligned}
& \text { (i) } \frac{1}{T\left(e^{-\pi \sqrt{n} / 2}\right)}-2+T\left(e^{-\pi \sqrt{n} / 2}\right)=2^{3 / 2} A_{4, n}, \\
& \text { (ii) } \frac{1}{T\left(e^{-\pi /(2 \sqrt{n})}\right)}-2+T\left(e^{-\pi /(2 \sqrt{n})}\right)=\frac{2^{3 / 2}}{A_{4, n}} .
\end{aligned}
$$

Proof Setting $q=\mathrm{e}^{-\pi \sqrt{n / 4}}$ in Theorem 3.1(iv) and employing the definition of $A_{k, n}$ with $k=4$, we arrive at (i). Replacing $n$ by $1 / n$ in (i) and simplifying using Lemma 2.1, we complete the proof of (ii).

Remark 5.6 From Theorem 5.5(i) and (ii) it is obvious that if we know explicit values of the parameter $A_{4, n}$ then explicit values of $T\left(e^{-\pi \sqrt{n} / 2}\right)$ and $T\left(e^{-\pi / 2 \sqrt{n}}\right)$ can easily be evaluated, respectively. For example, setting $n=1$ in Theorem 5.3(i), noting $A_{4,1}=1$ from Lemma 2.1 and solving the resulting equation, we evaluate

$$
T\left(e^{-\pi / 2}\right)=1+\sqrt{2}-\sqrt{2+2 \sqrt{2}} .
$$

A systematic study of the parameter $A_{k, n}$ for $k=4$ has not been undertaken and no other value of the parameter $A_{4, n}$ is evaluated in literature. So we devote the remainder of this section to evaluate some new explicit values of $A_{4, n}$ by using $P-Q$ theta-function identities established in Sect. 2.

Theorem 5.7 We have

$$
\begin{aligned}
& \text { (i) } A_{4,2}=1+\sqrt{1+\sqrt{2}} \\
& \text { (ii) } A_{4,4}=2+\sqrt{2}+\sqrt{2(4+3 \sqrt{2})}
\end{aligned}
$$

Proof (i) Setting $q=\mathrm{e}^{-\pi \sqrt{n / 4}}$ in Lemma 2.6 and employing the definition of $A_{k, n}$ with $k=4$ from (1.15), we obtain

$$
P=2^{3 / 2} A_{4, n} \quad \text { and } \quad Q=2^{3 / 2} A_{4,4 n} .
$$

Setting $n=1 / 2$ in (5.2) and simplifying using Lemma 2.1, we obtain

$$
P=\frac{2^{3 / 2}}{A_{4,2}} \quad \text { and } \quad Q=2^{3 / 2} A_{4,2}
$$

Employing (5.3) in (2.8) and simplifying, we deduce that

$$
A_{4,2}^{4}-4 A_{4,2}^{2}-4 \sqrt{2} A_{4,2}-2=0 .
$$

Solving (5.4) using Mathematica and noting $A_{4, n}$ has positive real value greater than unity, we arrive at (i).

(ii) Setting $n=1$ in (5.2), employing (2.8) and simplifying, we obtain

$$
A_{4,4}^{2}-(4+2 \sqrt{2}) A_{4,4}-(2+2 \sqrt{2})=0 .
$$

Solving (5.5) and choosing the appropriate root, we complete the proof of (ii).

Theorem 5.8 We have

$$
\begin{aligned}
& \text { (i) } A_{4,3}=\frac{1}{2}(2+\sqrt{2}+\sqrt{18+12 \sqrt{2})}) \text {, } \\
& \text { (ii) } A_{4,9}=5+3 \sqrt{2}+\sqrt{3(17+12 \sqrt{2})} \\
& +\sqrt{93+66 \sqrt{2}+314 \sqrt{3(17-12 \sqrt{2})}+222 \sqrt{6(17-12 \sqrt{2})} .}
\end{aligned}
$$


Proof (i) Setting $q=\mathrm{e}^{-\pi \sqrt{n / 4}}$ in Lemma 2.7 and employing the definition of $A_{k, n}$ with $k=4$ from (1.15), we obtain

$$
P=2^{3 / 2} A_{4, n} \quad \text { and } \quad Q=2^{3 / 2} A_{4,9 n} .
$$

Setting $n=1 / 3$ in (5.6) and simplifying using Lemma 2.1, we obtain

$$
P=\frac{2^{3 / 2}}{A_{4,3}} \quad \text { and } \quad Q=2^{3 / 2} A_{4,3} .
$$

Employing (5.7) in (2.14) and simplifying with the help of Mathematica, we deduce that

$$
A_{4,3}^{8}-12 A_{4,3}^{6}-24 \sqrt{2} A_{4,3}^{5}-46 A_{4,3}^{4}-24 \sqrt{2} A_{4,3}^{3}-12 A_{4,3}^{2}+1=0 .
$$

Solving (5.8) using Mathematica and choosing the appropriate root, we arrive at (i).

(ii) Setting $n=1$ in (5.6), employing in (2.14) and simplifying, we obtain

$$
A_{4,9}^{4}-(20+12 \sqrt{2}) A_{4,9}^{3}-(30+24 \sqrt{2}) A_{4,9}^{2}-(20+12 \sqrt{2}) A_{4,9}+1=0 .
$$

Solving (5.9) using Mathematica and choosing the appropriate root, we complete the proof of (ii).

Theorem 5.9 We have

$$
\text { (i) } \begin{aligned}
A_{4,5}= & \frac{1}{2}(3+\sqrt{2}+\sqrt{5(3+2 \sqrt{2})}) \\
& +\frac{1}{\sqrt{2}} \sqrt{(19+14 \sqrt{2}+2 \sqrt{5(3+2 \sqrt{2})}+3 \sqrt{10(3+2 \sqrt{2})})} \\
\text { (ii) } A_{4,25}= & 114+80 \sqrt{2}+3 \sqrt{5(577+408 \sqrt{2})} \\
+ & 2 \sqrt{12940+9150 \sqrt{2}+171 \sqrt{5(577+408 \sqrt{2})+120 \sqrt{10(577+408 \sqrt{2})}}} .
\end{aligned}
$$

Proof (i) Setting $q=\mathrm{e}^{-\pi \sqrt{n / 4}}$ in Lemma 2.8 and employing the definition of $A_{k, n}$ with $k=4$ from (1.15), we obtain

$$
P=2^{3 / 2} A_{4, n} \quad \text { and } \quad Q=2^{3 / 2} A_{4,25 n} .
$$

Setting $n=1 / 5$ in (5.10) and simplifying using Lemma 2.1, we obtain

$$
P=\frac{2^{3 / 2}}{A_{4,5}} \quad \text { and } \quad Q=2^{3 / 2} A_{4,5}
$$

Employing (5.11) in (2.19) and simplifying with the help of Mathematica, we deduce that

$$
\begin{aligned}
& A_{4,5}^{12}-70 A_{4,5}^{10}-320 \sqrt{2} A_{4,5}^{9}-1425 A_{4,5}^{8}-1920 \sqrt{2} A_{4,5}^{7}-3348 A_{4,5}^{6}-1920 \sqrt{2} A_{4,5}^{5} \\
& -1425 A_{4,5}^{4}-320 \sqrt{2} A_{4,5}^{3}-70 A_{4,5}^{2}+1=0 .
\end{aligned}
$$

Solving (5.12) using Mathematica and choosing the appropriate root, we arrive at (i).

(ii) Setting $n=1$ in (5.10), employing in (2.19) and simplifying, we obtain

$$
\left(1+y^{2}\right)\left(y^{4}+(456+320 \sqrt{2}) y^{3}-(674+480 \sqrt{2}) y^{2}-(456+320 \sqrt{2}) y+1\right)=0,
$$

where

$$
y=A_{4,25} .
$$

dividing second factor of (5.13) by $y^{2}$ and rearranging the terms, we obtain

$$
\left(y^{2}+\frac{1}{y^{2}}\right)+(456+320 \sqrt{2})\left(y+\frac{1}{y}\right)-(674+480 \sqrt{2})=0 .
$$


Equivalently,

$$
\left(z^{2}-2\right)-(456+320 \sqrt{2}) z-(674+480 \sqrt{2})=0
$$

where

$$
y+\frac{1}{y}=z .
$$

Solving (5.16) and choosing the positive real root greater than unity, we obtain

$$
z=2(114+80 \sqrt{2}+3 \sqrt{5(577+408 \sqrt{2})}) .
$$

Employing (5.18) in (5.17), solving for $y=A_{4,25}$, and choosing the appropriate root, we complete the proof of (ii).

\section{General theorems for the explicit evaluations of $W(q)$}

In this section we prove general theorems for the explicit evaluations of the continued fraction $W(q)$.

Theorem 6.1 If $s_{4, n}$ is as defined (1.16), then

$$
\frac{1}{W\left(e^{-\pi \sqrt{n} / 4}\right)}+4 W\left(e^{-\pi \sqrt{n} / 4}\right)=4 s_{4, n}^{4} .
$$

Proof Setting $q:=\mathrm{e}^{-\pi \sqrt{n} / 2}$ in Theorem 4.1(i) and employing the definition of $s_{4, n}$, we complete the proof.

Remark 6.2 From Theorem 6.1 it is clear that if we know the explicit values of $s_{4, n}$ then explicit values of $W\left(e^{-\pi \sqrt{n} / 4}\right)$ can easily be evaluated. For example, setting $n=8$ in Theorem 6.1, employing the value $s_{4,8}=(1+\sqrt{2})^{1 / 4}$ from [3, p. 276, Corollary 3.3.(iv)], and solving the resulting equation, we evaluate

$$
W\left(e^{-\pi / \sqrt{2}}\right)=(1+\sqrt{2}-\sqrt{2(1+\sqrt{2})}) / 2 .
$$

Theorem 6.3 If $J_{n}$ is as defined in (1.17), then

$$
\begin{aligned}
& \text { (i) } \frac{1}{W\left(\mathrm{e}^{-\pi \sqrt{n} / 2}\right)}-4 W\left(\mathrm{e}^{-\pi \sqrt{n} / 2}\right)=4 J_{n}^{4}, \\
& \text { (ii) } \frac{1}{W\left(\mathrm{e}^{-\pi /(2 \sqrt{n})}\right)}-4 W\left(\mathrm{e}^{-\pi /(2 \sqrt{n})}\right)=\frac{4}{J_{n}^{4}} .
\end{aligned}
$$

Proof Setting $q=\mathrm{e}^{-\pi \sqrt{n}}$ in Theorem 4.1(ii)and employing the definition of $J_{n}$, we arrive at (i). To prove (ii), we replace $n$ by $1 / n$ in part (i) and use Lemma 2.2.

Remark 6.4 From Theorem 6.3(i) and (ii) it is clear that if we know explicit values of $J_{n}$ then explicit values of $W\left(e^{-\pi \sqrt{n} / 2}\right)$ and $W\left(e^{-\pi /(2 \sqrt{n})}\right)$ can easily be calculated, respectively. Baruah and Saikia [2] evaluated $J_{n}$ for $n=1,2,3,4,5,6,7,8,9,10,11,13,15,16,17,18,19,23,25,31,36$, and 49. Saikia [16] also evaluated $J_{n}$ for $n=15,5 / 3,21,7 / 3,33$, and 11/3. For example, setting $n=3$ in Theorem 6.3(i) and (ii), employing the value of $J_{3}$ and solving the resulting equations, we evaluate

$$
W\left(e^{-\pi \sqrt{3} / 2}\right)=(-2-\sqrt{3}+\sqrt{8+4 \sqrt{3}}) / 2
$$

and

$$
W\left(e^{-\pi /(2 \sqrt{3})}\right)=(-1+\sqrt{8+4 \sqrt{3}})(2-\sqrt{3}) / 2,
$$

respectively. 
Theorem 6.5 We have

$$
\begin{aligned}
& \text { (i) } \frac{1}{W\left(e^{-\pi \sqrt{n / 2}}\right)}-4+4 W\left(e^{-\pi \sqrt{n / 2}}\right)=2^{5 / 2} A_{2, n}^{2}, \\
& \text { (ii) } \frac{1}{W\left(e^{-\pi / \sqrt{2 n}}\right)}-4+4 W\left(e^{-\pi / \sqrt{2 n}}\right)=\frac{2^{5 / 2}}{A_{2, n}^{2}} .
\end{aligned}
$$

Proof Setting $q=\mathrm{e}^{-\pi \sqrt{n / 2}}$ in Corollary 4.3(ii) and employing the definition of $A_{k, n}$ with $k=2$, we arrive at (i). To prove (ii), we replace $n$ by $1 / n$ in (i) and simplify using Lemma 2.1 .

Remark 6.6 From Theorem 6.5(i) and (ii) it is clear that if we know explicit values of $A_{2, n}$ then explicit values of $W\left(e^{-\pi \sqrt{n / 2}}\right)$ and $W\left(e^{-\pi / \sqrt{2 n}}\right)$ can be evaluated, respectively. For example, setting $n=2$ in Theorem 6.5(i) and (ii), employing the value $A_{2,2}=\sqrt{2+\sqrt{2}}$ from [13, p. 114, Theorem 5.2(i)] and solving the resulting equations, we evaluate

$$
W\left(e^{-\pi}\right)=(3+2 \sqrt{2}-2 \sqrt{4+3 \sqrt{2}}) / 2
$$

and

$$
W\left(e^{-\pi / 2}\right)=(\sqrt{2}-1) / 2
$$

respectively.

Open Access This article is distributed under the terms of the Creative Commons Attribution 4.0 International License (http:// creativecommons.org/licenses/by/4.0/), which permits unrestricted use, distribution, and reproduction in any medium, provided you give appropriate credit to the original author(s) and the source, provide a link to the Creative Commons license, and indicate if changes were made.

\section{References}

1. Andrews, G.E.: On $q$-difference equations for certain well-poised basic hypergeometric series. Q. J. Math. (Oxford) 19, 433447 (1968)

2. Baruah, N.D.; Saikia, N.: Modular equations and explicit values of Ramanujan-Selberg continued fraction. Int. J. Math. Math. Sci. Article ID 54901, 2006, 1-15 (2006)

3. Baruah, N.D.; Saikia, N.: Explicit evaluations of Ramanujan-Göllnitz-Gordon continued fraction. Monatsh. Math. 154, 271-288 (2008)

4. Baruah, N.D.; Bora, J.; Saikia, N.: Some new proofs of modular relations for the Göllnitz-Gordon functions. Ramanujan J. 15, 281-30 (2008)

5. Berndt, B.C.: Ramanujan's Notebooks, Part III. Springer, New York (1991)

6. Berndt, B.C.: Flowers which we cannot yet see growing in Ramanujan's garden of hypergeometric series, elliptic functions, and q's, in Special Functions 2000: Current Perspective and Future Directions. In: Bustoz, J.; Ismail, M.E.H.; Suslov, S.K. (eds.), Kluwer, Dordrecht. pp. 61-85 (2001)

7. Chan, H.H.; Huang, S.-S.: On the Ramanujan-Göllnitz-Gordon continued fraction. Ramanujan J. 1, 75-90 (1997)

8. Göllnitz, H.: Partitionen mit Differenzenbedingungen. J. Reine Angew. Math. 225, 154-190 (1967)

9. Gordon, B.: Some continued fractions of Rogers-Ramanujan type. Duke Math. J. 32, 741-748 (1965)

10. Ramanathan, K.G.: Ramanujan's continued fraction. Indian J. Pure Appl. Math. 16, 695-724 (1985)

11. Ramanujan, S.: Notebooks (2 volumes). Tata Institute of Fundamental Research, Bombay (1957)

12. Saikia, N.: On modular identities of Ramanujan-Göllnitz-Gordon continued fraction. Far East J. Math. Sci. 54(1), 6579 (2011)

13. Saikia, N.: A new parameter for Ramanujan's theta-fucntions and explicit values. Arab. J. Math. Sci. 18, 105-119 (2012)

14. Saikia, N.: Modular identities and explicit evaluations of a continued fraction of Ramanujan. Int. J. Math. Math. Sci. 2012(694251), 1-10 (2012)

15. Saikia, N.: A new continued fraction of Ramanujan, its modular identities and explicit evaluations. Afr. Mat. 26, 407417 (2015)

16. Saikia, N.: Some new explicit values of Ramanujan-Selberg continued fraction (Submitted)

17. Saikia, N.: General theorem for explicit evaluations and reciprocity theorems for Ramanujan-Göllnitz-Gordon continued fraction. Kyungpook Math. J. 55, 983-996 (2015)

18. Vasuki, K.R.; Srivatsa Kumar, B.R.: Certain identities for Ramanujan-Göllnitz Gordon continued fraction. J. Comput. Appl. Math. 187, 87-95 (2006)

19. Yuttanan, B.: New properties for the Ramanujan-Göllnitz-Gordon continued fraction. Acta Arith. 151(3), 293-310 (2012)

20. Yi, J.: Construction and Application of Modular Equations. Ph. D. Thesis, University of Illinois at Urbana Champaign (2004) 